
\title{
25 Research Square \\ The Possibility of Analyzing Endometrial Receptivity Using Cells From Embryo Transfer Catheters
}

\section{Kaori Goto}

St. Luke Clinic, 1-4-5, Higashi Omichi, Oita

Yasushi Kawano ( $\nabla$ kawayas@oita-u.ac.jp )

Oita University https://orcid.org/0000-0001-8480-0168

\section{Takafumi Utsunomiya}

St. Luke Clinic, 1-4-5, Higashi Omichi, Oita

\section{Hisashi Narahara}

Oita University

\section{Research}

Keywords: Endometrial receptivity, ET catheter, collection method, mRNA expression, protein expression

Posted Date: October 26th, 2020

DOI: https://doi.org/10.21203/rs.3.rs-93703/v1

License: (c) (1) This work is licensed under a Creative Commons Attribution 4.0 International License.

Read Full License

Version of Record: A version of this preprint was published at Reproductive Sciences on June 3rd, 2021. See the published version at https://doi.org/10.1007/s43032-021-00645-9. 


\section{Abstract}

Background: Endometrial receptivity issues represent a potential source of implantaion failure. It is very important to investigate the expression of endometrial receptive markers in the endometrium during implantation. Therefore, we examined whether it would be possible to analyze endometrial receptivity using cells from embryo transfer catheters. In addition, we analyzed the relationship between the gene expression profile associated with pregnancy from endometrial cells taken during embryo transfer.

Methods: A total of 88 cycles from 88 consenting patients were enrolled in this study. The tip of the embryo transfer (ET) catheter was cut and immersed in a dedicated reagent. Confirmation of cell distribution was carried out using a Papanicolaou stain $(n=6)$ and immunocytochemistry $(n=3)$. Protein expression was carried out by immunocytochemistry $(n=12)$. Total RNA was extracted, and the expression of endometrial receptive markers (estrogen receptor a, progesterone receptor, and homeobox A10) were analyzed using quantitative reverse transcription polymerase chain reaction $(n=67)$. we analyzed the relationship between the gene expression profile associated with pregnancy from endometrial cells.

Results: Samples collected from the ET catheter showed clear staining for endometrial cells. Most of the cells were endometrial epithelial cells. Cervical cells were not included. The protein expression of endometrial receptive markers in cells was also confirmed. Three genes were analyzed that are associated with endometrial receptivity. Progesterone receptor expression was 1.5-fold and homeobox A10 expression was 2-fold higher in patients who became non-pregnant group, compared to the pregnant group. Both increases were statistically significant $(p<0.05)$. Estrogen receptor a expression tended to be higher in the non-pregnant group, but there was no significant difference.

Conclusion: Our results suggest that endometrial receptivity can be evaluated using cells obtained from the ET catheter. This method may be useful for elucidating the cause of implantation failure by comparing a receptive and non-receptive endometrium at the time of ET.

\section{Introduction}

Implantation of the blastocyst into the uterine endometrium remains a significant limiting step for success in in-vitro fertilization and embryo transfer (IVF-ET) [1]. It is important to gain an understanding of the fertile endometrial physiology that can successfully support implantation; and the subsequent maintenance of pregnancy and placentation. We previously reported that endometrial cell function is changed by decidualization in association with increasing protease-activated receptor (PAR) -1 expression [2]. The up-regulation of PAR-1 in decidualized endometrial stromal cells may have some effect on pregnancy.

In human endometrial epithelial cells, gene expression of cytokeratin (CK) 15 is reported to decrease in a homeobox A (HOXA) 10- dependent fashion [3]. It is reported that dramatic changes in cellular architecture are necessary to achieve the secretory changes in the endometrial epithelium that bring 
about the implantation window. In order to improve the implantation rate, we have assessed an embryo's ability to develop into a good-quality blastocyst using time-lapse imaging and the oxygen consumption rate [4]. Embryos with good morphology showed a higher euploidy rate compared with lower-quality embryos [5]. Even euploid, morphologically normal blastocysts fail to implant in about one-third of transfers $[6,7]$. This failure rate for the implantation of euploid embryos may suggest a non-embryonic source of implantation failure, with endometrial receptivity issues representing another potential cause. It has been recognized that, for successful implantation, there must be developmental synchrony between the embryo and the endometrium [8].

Recently, the endometrial receptivity array (ERA) was developed as an objective molecular dating method to accurately and reproducibly identify endometrial receptivity status from endometrial tissue samples [9]. There have been several reports concerning the effectiveness and significance of the ERA [10-12]. One limitation of endometrial gene expression studies has been the need to use cells collected by endometrial biopsy for profiling in previous cycle [13]. Sequential invasive sampling of the endometrium during a single cycle can result in wound-related confounding and can alter the biomarker candidates. Temporal and regional gene expression variations found within the endometrium may make selecting the ideal conditions for embryo implantation difficult. Therefore, we tried to collect endometrial cells in a minimally invasive way from the implantation site of an ET cycle. The purpose of this study is to clarify the possibility to analyze endometrial receptivity using these cells from an ET catheter.

\section{Methods}

\section{Patients}

A total of 88 cycles were studied from 88 consenting patients enrolled in this study who were treated for infertility at St. Luke Clinic during the period from December 2019 to June 2020. Informed consent was obtained from all patients in accordance with a protocol reviewer and approved by the institutional ethical review board of Oita University, Yufu, Japan.

\section{Endometrial cell isolation}

ET was performed on Day 5 with confirmation of uterine conformation as well as evaluation of the endometrium for a tight trilaminar structure (over $7 \mathrm{~mm}$ ) using transvaginal ultrasonography. At that point, progesterone had already been used for 5 days. Once these conditions were identified, a flexible guide (KITAZATO, Fuji, Japan) for the ET catheter was introduced through the cervix, ensuring contact with the endometrium with ultrasonography guidance. After the guide position was determined, the stylet was removed. After ET, the catheter was pulled back into the guide and taken out. The tip of the catheter was cut by about $3 \mathrm{~cm}$ and immersed in $350 \mu$ of lysis buffer for RNA extraction. In order to confirm the cytological determination of endometrial cells or the expression of endometrial receptive markers, the tip of the catheter was cut by about $3 \mathrm{~cm}$ and immersed into the fixative.

\section{Cell distribution and immunocytochemistry}


The endometrial cells were attached to a microscope slide and then stained with Papanicolaou stain (Papa) according to standard procedures. The cells were diagnosed as endometrial cells with a cytotechnologist (The International Academy of Cytology) $(n=6)$. The cytokeratin (AE1/AE3) $(n=3)$ and the expressions of putative endometrial receptive markers including estrogen receptor a $(E R a, n=3)$, progesterone receptor $(P R, n=3)$, and HOXA $10(n=3)$ were evaluated by immunocytochemistry. The sections were treated in ethanol, and endogenous peroxidase was blocked with $3 \% \mathrm{H}_{2} \mathrm{O}_{2}$. After a 60minute incubation with Protein Block Serum-Free (X0909 Dako, Santa Clara, USA), the sections were incubated for 60 minutes at room temperature with primary antibody (Table 1). The sections were then incubated with MAX-PO (MULTI). MAX-PO is a labeled polymer in which a peroxidase and a second antibody made into Fab are bonded to an amino acid polymer. Hematoxylin was used for counterstaining. Negative control sections were incubated without the primary antibody $(n=3)$. Images were taken under a confocal microscope (Keyence BZ-9000, Osaka, Japan).

\section{RNA extraction and quantitative reverse transcription polymerase chain reaction (RT-qPCR)}

Total RNA was extracted from the cellular suspension using the RNeasy Micro Kit (Qiagen, Hilden, Germany) according to the manufacturer's instructions ( $n=67)$.

RT was performed using the iScript ${ }^{\mathrm{TM}}$ Advanced cDNA Synthesis Kit for RT-qPCR (BIO-RAD, Hercules, USA). All total RNAs were reverse transcribed in a $20-\mu$ l volume. The real-time-qPCR was carried out in duplicate with a LightCycler 480 (Loche Diagnosis, Penaberg, Germany) according to the manufacturer's instructions. The PCR primers were purchased from Applied Biosystems (Carlsbad, CA, USA) (Table 2). The expression of mRNA was normalized to RNA loading for each sample using GAPDH mRNA as an internal standard. These expression levels were calculated by the $\triangle \triangle C T$ method.

\section{Statistical analysis}

Data are presented as the mean $\pm S D$ of duplicate samples and are presented as folds relative to the corresponding controls, as the mean \pm SD values were analyzed by Bonferroni-Dunn test. A level of $p<0.05$ was considered significant. The confidence intervals with $p$-values for multiple statistical analyses are at the $95 \%$ level.

\section{Results}

\section{Collection and characterization of the endometrial cells}

After ET, the tip of the catheter was cut by about $3 \mathrm{~cm}$ and immersed in the fixative. Cells attached to the catheter were evaluated for endometrial cells. The morphological evaluation by Papa staining suggested that endometrial epithelial cells were detected as the majority of cells and that they were not contaminated with cervical cells from the cervix neck (Fig. 1a and b). These cells were confirmed and diagnosed as endometrial cells with a cytotechnologist. Additionally, immunocytochemical analysis revealed that positive staining for AE1/AE3 was detected in about $90 \%$ of the endometrial cells (Fig. 1c 
and d). Immunocytochemical assays were performed by at least two researchers in an operator-blinded manner.

\section{Expression of endometrial receptive markers}

In order to investigate the protein expression of endometrial receptive markers in cells attached to the catheter, the expressions of ERa, PR, and HOXA 10 were confirmed and evaluated by immunocytochemistry in endometrial cells (Fig. 2).

\section{mRNA expression of endometrial receptive markers}

In order to investigate the mRNA expression of endometrial receptive markers in cells attached to the catheter, the variation of mRNA expression was evaluated. When total RNA was extracted from the cellular suspension, the concentrations of extracted RNA were 3.7 to $43.4 \mathrm{ng} / \mu \mathrm{l}$ (data was not shown). Three genes (ERa, PR, HOXA 10) were evaluated that are associated with endometrial receptivity, Fig. 3 shows the distribution of the endometrial receptive markers. When the expression in the pregnant group was 1 , the expression in the non-pregnant group was $1.3,1.5$, and 2 -fold, respectively, and the PR and HOXA10 mRNA expressions were significantly higher in the non-pregnant group $(p<0.05)$ (Fig. 4).

\section{Discussion}

Endometrial receptivity has been recognized as a potential source of implantation of embryo. Histological evaluations have been regarded as the standard for the clinical diagnosis of endometrial abnormalities [14]. Recently, many studies have investigated the potential of proteomic analysis to characterize the expression patterns of physiologically active substances on proliferative and secretory endometrium [1517]. The identification of gene expression profiles has led to a differential analysis of receptive and nonreceptive patterns in endometrial signaling. The use of these expression patterns from endometrial tissues has been demonstrated to be an accurate and reproducible dating method to identify endometrial receptivity status. In the present study, our experimental results demonstrated that cells that came in contact with a catheter specifically positioned on the surface of the endometrium could provide insight into the level of endometrium receptivity. Indeed, using the cells attached to the catheter, we were able to evaluate the expression of genes associated with endometrial receptivity, and their expression differed by pregnancy outcome. It may be possible to identify the optimal luteal management or ET-timing by analyzing this difference in expression. Furthermore, not only gene expression but also protein expression could be confirmed by using this method.

By profiling the transcriptome of 238 genes that are expressed at different stages of the endometrial cycle, the ERA was developed as a means of personalizing ET timing. It has been demonstrated to be reproducible in patients across multiple menstrual cycles and to be more accurate than histological analysis in defining the optimal window of implantation (WOI) [9]. Although this method has significant benefits, it does invade the endometrium, and it may not be able to reveal dell information related to the transfer cycle. In addition, the cost of measurement is high. On the other hand, our method allowed for 
current cycle analysis, without an additional invasive procedure. Moreover, due to the nature of ET, this sample was taken without altering the ET. An important problem that we faced was that we can only collect a small amount of cells, which limits the potential number of endpoints to investigate. Therefore, the next aim of this study was to narrow down the gene profile at implantation that would have a greater potential of leading to pregnancy.

ERa, PR, and HOXA 10 have been analyzed as endometrial receptive markers. These genes have been reported to be associated with implantation [18-22]. In our results, progesterone receptor expression was 1.5-fold and HOXA10 expression was 2-fold significantly higher in patients who became non-pregnant group. Gene expression of HOXA 10 was reportedly is increased in a progesterone-dependent fashion in human endometrium [23]. On the other hand, gene expression of CK 15 was decreased in a HOXA10dependent fashion in human endometrium epithelial cells [3]. Alterations in CK15 could facilitate these cytoskeletal changes, ultimately promoting endometrial receptivity. From our results, higher expression of HOXA10 in non-pregnant group compared with pregnant group suggested that excessive uptake of progesterone may contribute to modulate an excessive increase in HOXA10, resulting to low decrease in cytokeratin. Therefore, increasing HOXA10 may cause to be difficult to implantation by making cell structure fragile. In other report, colony stimulating factor-1 and LIF expression in endometrial cells isolated from current cycle cannula cells showed associations with increasing endometrial receptivity and pregnancy [20]. Prior to that, it was reported that a significant proportion of patients with a history of failure of the implantation of a euploid embryo had a displaced WOI as detected by the ERA [24]. For these patients, personalized ET using a modified progesterone administration protocol may improve the outcomes of subsequent euploid ET. Our new method will be able to analyze the condition of the endometrium in order to optimize the luteal management or ET-timing. It is important to determine the gene profile at implantation that would have the greatest potential of leading to pregnancy.

This procedure that we have established made it possible to conduct analysis in the current cycle, without an additional invasive procedure. Moreover, the cells attached to the catheter were revealed information about the transfer site without altering the endometrium function, and with minimal contamination from other cells that came in contact with the catheter. In addition, this information may lead to ameliorate the endometrial conditions with next implantation, even if implantation of this cycle is failed. Taken together, this procedure could prove to be a reliable method for detecting genes to assess endometrial receptivity.

A limitation of our study is that we examined slight gene expressions in cells obtained from the surface of endometrium. The remaining question is whether any other genes are present with modulating the implantation or the perturbation of gene expression could cause implantation failure in endometrium. Further study concerning the comprehensive analysis between pregnant and non-pregnant will be needed.

In conclusion, our findings have revealed that our method can be used to take a sample of the endometrium, and this method does not affect the condition of ET nor is it invasive. This method can evaluate the state of the endometrium at the time of ET and may contribute to the improvement of implantation. 


\section{Abbreviations}

CK

Cytokeratin; ERa:Estrogen receptor a; ERA:Endometrial receptivity array; ET:Embryo transfer; HOXA 10:Homeobox A 10; IVF-ET:in-vitro fertilization and embryo transfer; Papa:Papanicolaou stain; PAR1:Protease-activated receptor-1; PR:Progesterone receptor; RT-qPCR:reverse transcription polymerase chain reaction;

\section{Declarations}

\section{Ethics approval and consent to participate}

The study design was approved by the appropriate ethics committee of Oita University, Yufu, Japan.

\section{Consent for publication}

Not applicable.

\section{Availability of data and materials}

Literature search results are available from the authors on reasonable request

\section{Competing interests}

The authors declare that they have no competing interests.

\section{Funding}

This research was supported in part by a Grant-in-Aid (No. JP 20 K18193 to K.G.) for Specific Research from the Ministry of Education, Sports, Science, and Culture of Japan.

\section{Authors' contributions}

K.G. and Y.K. contributed to the study's design, forming conclusions and manuscript writing. K.G. and T.U. contributed to collect samples. K.G. contributed RT-PCR and immunocytochemical analysis. T.U.: data interpretation; H.N.: manuscript correction. All authors read and approved the final manuscript.

\section{Acknowledgments}

The authors are very grateful to a cytotechnologist for the diagnosis of cells.

\section{References}

1. Dekel N, Gnainsky Y, Granot I, Racicot K, Mor G. The role of inflammation for a successful implantation. Am J Reprod Immunol. 2014; 72:141-7. 
2. Goto K, Kawano Y, Utsunomiya T, Narahara H. Decidualization modulates a signal transduction system via protease-activated receptor-1 in endometrial stromal cells. Am J Reprod Immunol doi: 10.1111/aji.13036.

3. Kallen AN, Haines K, Taylor HS. HOXA10 Regulates expression of cytokeratin 15 in endometrial epithelial cytoskeletal remodeling. Reprod Sci.2014; 21: 892-897.

4. Goto K, Kumasako Y, Koike M, Kanda A, Kido K, Nagaki M, Otsu E, Kawabe F, Kai Y, Utsunomiya T. Prediction of the in vitro developmental competence of early-cleavage-stage human embryos with time-lapse imaging and oxygen consumption rate measurement. Reprod Med Biol. 2018; 17: 289296.

5. Capalbo A, Rienzi L, Cimadomo D, Maggiulli R, Elliott T, Wright G, Nagy ZP, Ubaldi FM. Correlation between standard blastocyst morphology, euploidy and implantation: an observational study in two centers involving 956 screened blastocysts. Hum Reprod.2014; 29: 1173-81.

6. Yang Z, Liu J, Collins GS, Salem SA, Liu X, Lyle SS, Peck AC, Sills ES, Salem RD. Selection of single blastocysts for fresh transfer via standard morphology assessment alone and with array CGH for good prognosis IVF patients: results from a randomized pilot study. Mol Cytogenet. 2012; 5:24.

7. Forman EJ, Hong KH, Ferry KM, Tao X, Taylor D, Levy B, Treff NR, Scott RT Jr. In vitro fertilization with single euploid blastocyst transfer: a randomized controlled trial. Fertil Steril. 2013; 100: 100-7.

8. Utsunomiya $\mathrm{T}$, Ito $\mathrm{H}$, Hirai K, Otsu E, Watanabe H, Mori T. Developmentallyretarded frozen blastocysts can be rescued by synchronizing culture prior to transfer. Reprod Biomed Online. 2006;12: 622-9.

9. Díaz-Gimeno P, Horcajadas JA, Martínez-Conejero JA, Esteban FJ, Alamá P, Pellicer A, Simón C. A genomicdiagnostic tool for human endometrial receptivity based on the transcriptomic signature. Fertil Steril. 2011; 95:50-60.

10. Ruiz-Alonso M, Blesa D, Díaz-Gimeno P, Gómez E, Fernández-Sánchez M, Carranza F, Carrera J, Vilella F, Pellicer A, Simón C. The endometrial receptivity array for diagnosis and personalized embryo transfer as a treatment for patients with repeated implantation failure. Fertil Steril. 2013; 100: 81824.

11. Ruiz-Alonso M, Galindo N, Pellicer A, Simón C. What a difference two days make: "personalized" embryo transfer (pET) paradigm: a case report and pilot study. Hum Reprod. 2014; 29: 1244-7.

12. Patel JA, Patel AJ, Banker JM, Shah SI, Banker MR. Personalized embryo transfer helps in improving in vitrofertilization/ICSI outcomes in patients with recurrent implantation failure. J Hum Reprod Sci. 2019; 12: 59-66.

13. Chan C, Virtanen C, Winegarden NA, Colgan TJ, Brown TJ, Greenblatt EM. Discovery of biomarkers of endometrial receptivity through a minimally invasive approach: a validation study with implications for assisted reproduction. Fertil Steril. 2013; 100: 810-7.

14. Noyes R W, Hertig A T, Rock Dating the endometrial biopsy. Am J Obstet Gynecol. 1975; 122:262-3.

15. Hernández-Vargas $P$, Muñoz M, Domínguez F. Identifying biomarkers for predicting successful embryo implantation: applying single to multi-OMICs to improve reproductive outcomes. Hum 
Reprod Update.2020; 26: 264-301.

16. Matorras R, Quevedo S, Corral B, Prieto B, Exposito A, Mendoza R, Rabanal A, Diaz-Nuñez $M$, Ferrando M, Elortza F, Ametzazurra A, Nagore D. Proteomicpattern of implantative human endometrial fluid in in vitro fertilization cycles. Arch Gynecol Obstet. 2018; 297: 1577-1586.

17. Koot YE, van Hooff SR, Boomsma CM, van Leenen D, Groot Koerkamp MJ, Goddijn M, Eijkemans MJ, Fauser BC, Holstege FC, Macklon NS. An endometrial gene expression signature accurately predicts recurrent implantation failure after IVF. Sci Rep.2016; 6: 19411.

18. CritchleyHO, Saunders Hormone receptor dynamics in a receptive human endometrium. Reprod Sci 2009; 16:191-199.

19. Hu M, Li J, Zhang Y, Li X, Brännström M, Shao LR, Billig H. Am J Transl Res. Endometrialprogesterone receptor isoforms in women with polycystic ovary syndrome. 2018; 10:2696-2705.

20. Camargo-Díaz F, García V, Ocampo-Bárcenas A, González-Marquez H, López-Bayghen E. Colony stimulating factor-1 and leukemia inhibitor factor expression from current-cycle cannula isolated endometrial cells are associated with increased endometrial receptivity and pregnancy. BMC Women's Health. 2017; 22; 17:63.

21. Godbole G, Suman P, Malik A, Galvankar M, Joshi N, Fazleabas A, Gupta SK, Modi D. Decreasein expression of HOXA10 in the decidua after embryo implantation promotes trophoblast invasion. 2017; 158:2618-2633.

22. Singh H, Nardo L, Kimber SJ, Aplin JD. Early stages of implantation as revealed by an in vitro model. 2010; 139:905-914.

23. L Ma, G V Benson, H Lim, S K Dey, R L Maas. Abdominal B (AbdB) Hoxa genes: regulation in adult uterus by estrogen and progesterone and repression in müllerian duct by the synthetic estrogen diethylstilbestrol (DES). Dev Biol. 1998; 197:141-154.

24. Tan J, Kan A, Hitkari J, Taylor B, Tallon N, Warraich G, Yuzpe A, Nakhuda G. The roleof the endometrial receptivity array (ERA) in patients who have failed euploid embryo transfers. J Assist Reprod Genet. 2018; 35:683-692.

\section{Tables}

Due to technical limitations, table 1,2 is only available as a download in the Supplemental Files section.

\section{Figures}




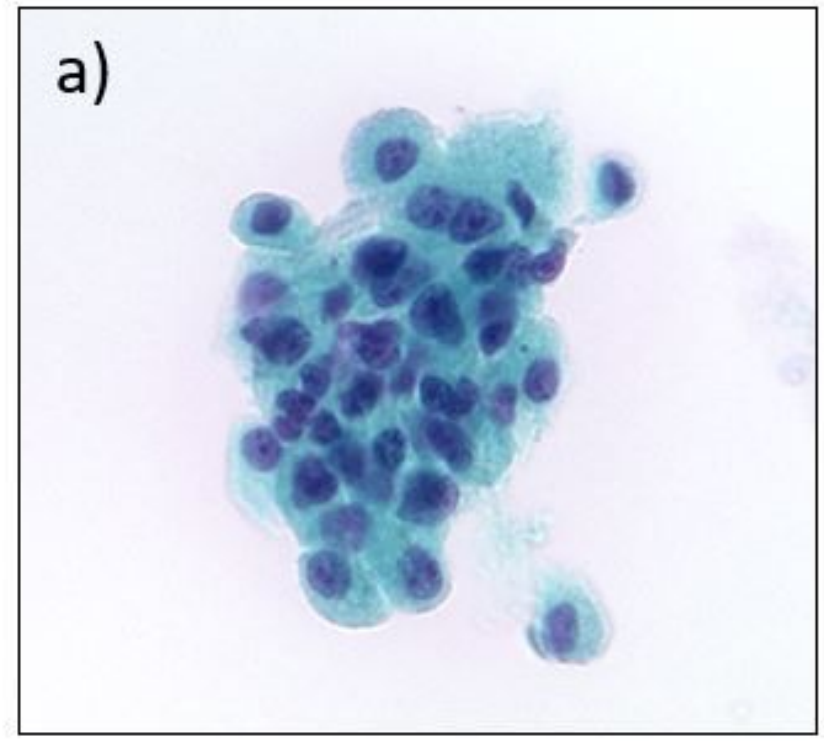

\section{b)}
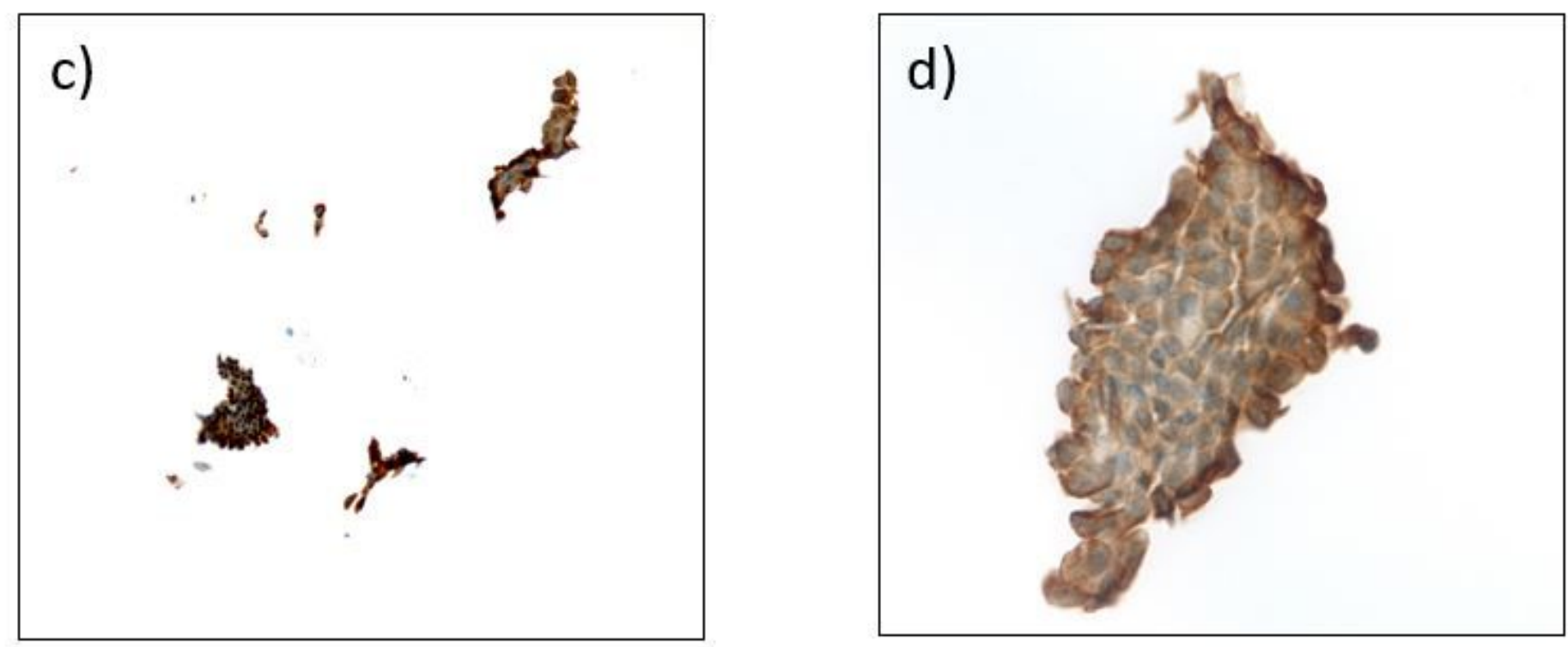

Figure 1

Endometrial cells attached to the catheter during embryo transfer. a) and b) Cells that attached to the catheter were stained by papanicolaou stain $(n=3), c)$ and d) were stained by immunocytochemical staining of AE1/AE3, then visualized with a Keyence BZ-9000 microscope ( $n=3)(a, b, d: 400 x, c: 200 x)$. 
$\mathrm{ER} \alpha$
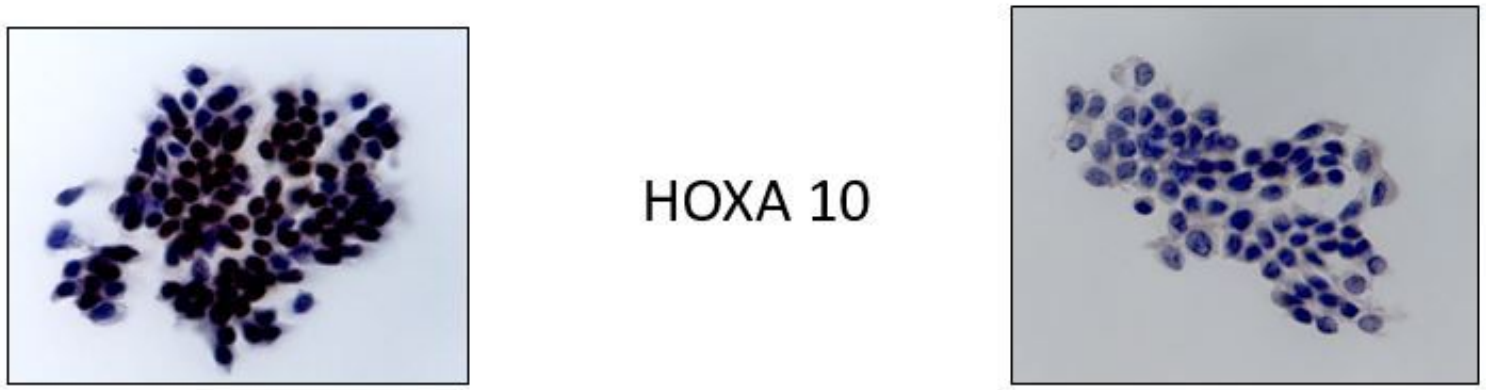

PR

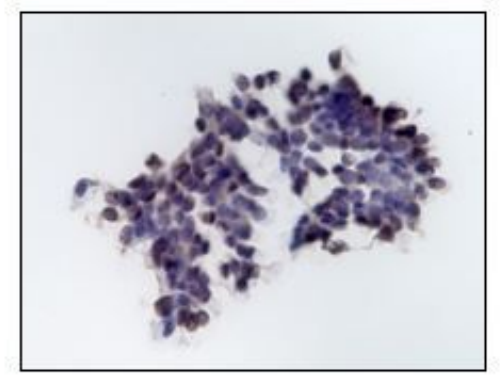

\section{HOXA 10}

Negative control

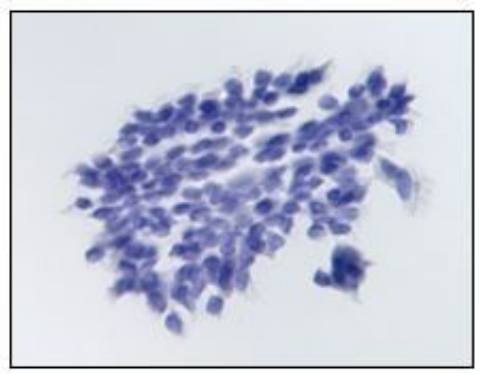

\section{Figure 2}

Expression of putative endometrial receptive markers in the human luteal-phase endometrium. Cells that attached to the catheter were stained using immunocytochemistry (ERa, PR, HOXA10, negative control; $n=3)$, then visualized with a Keyence BZ-9000 microscope (400x). 


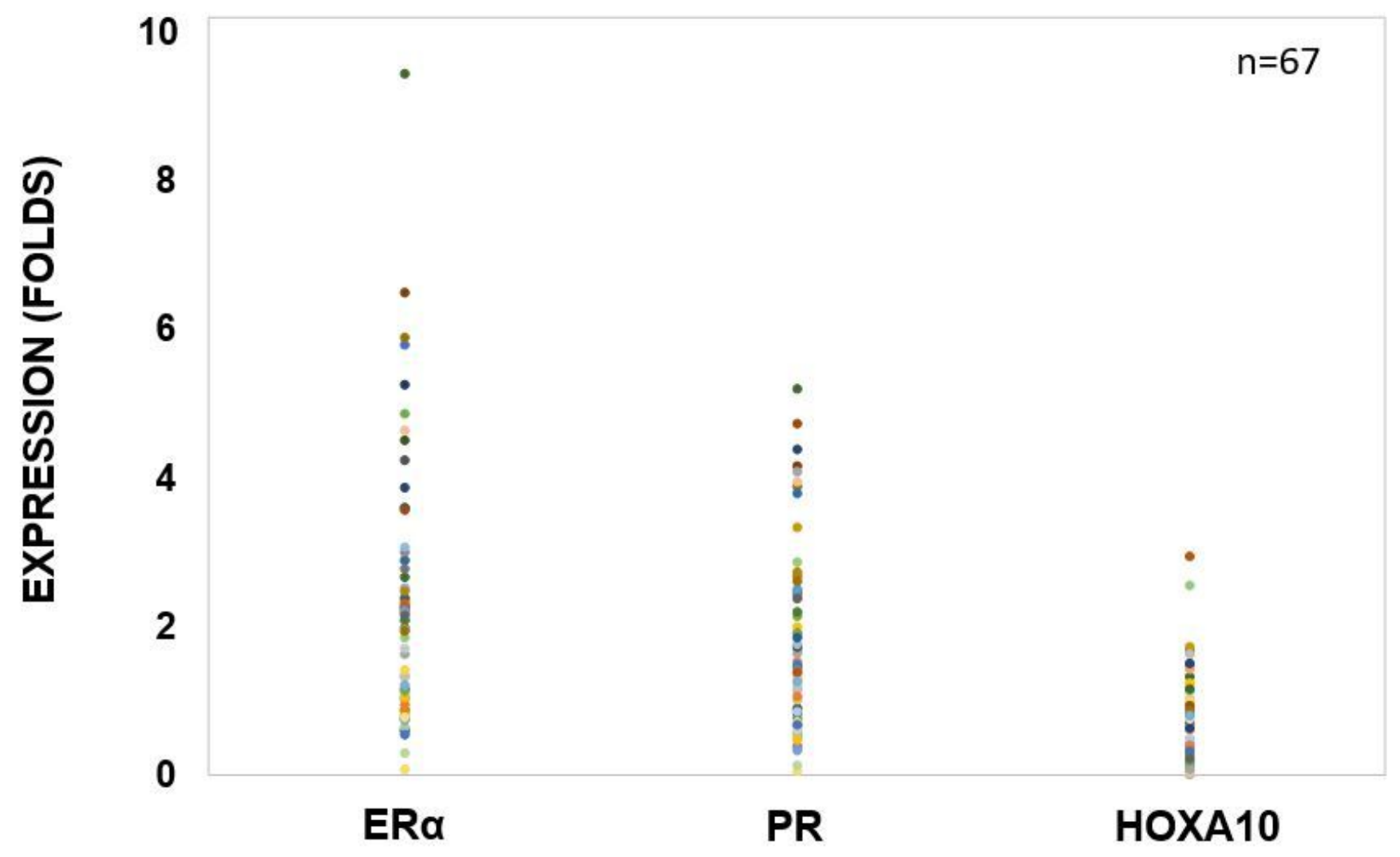

Figure 3

Distribution of the endometrial receptive markers. Total RNA was collected from 67 patient. Total RNA was extracted from the cells that attached to the catheter and subjected to RT-qPCR to determine the mRNA level. The expression of ERa, PR and HOXA10 was determined. 


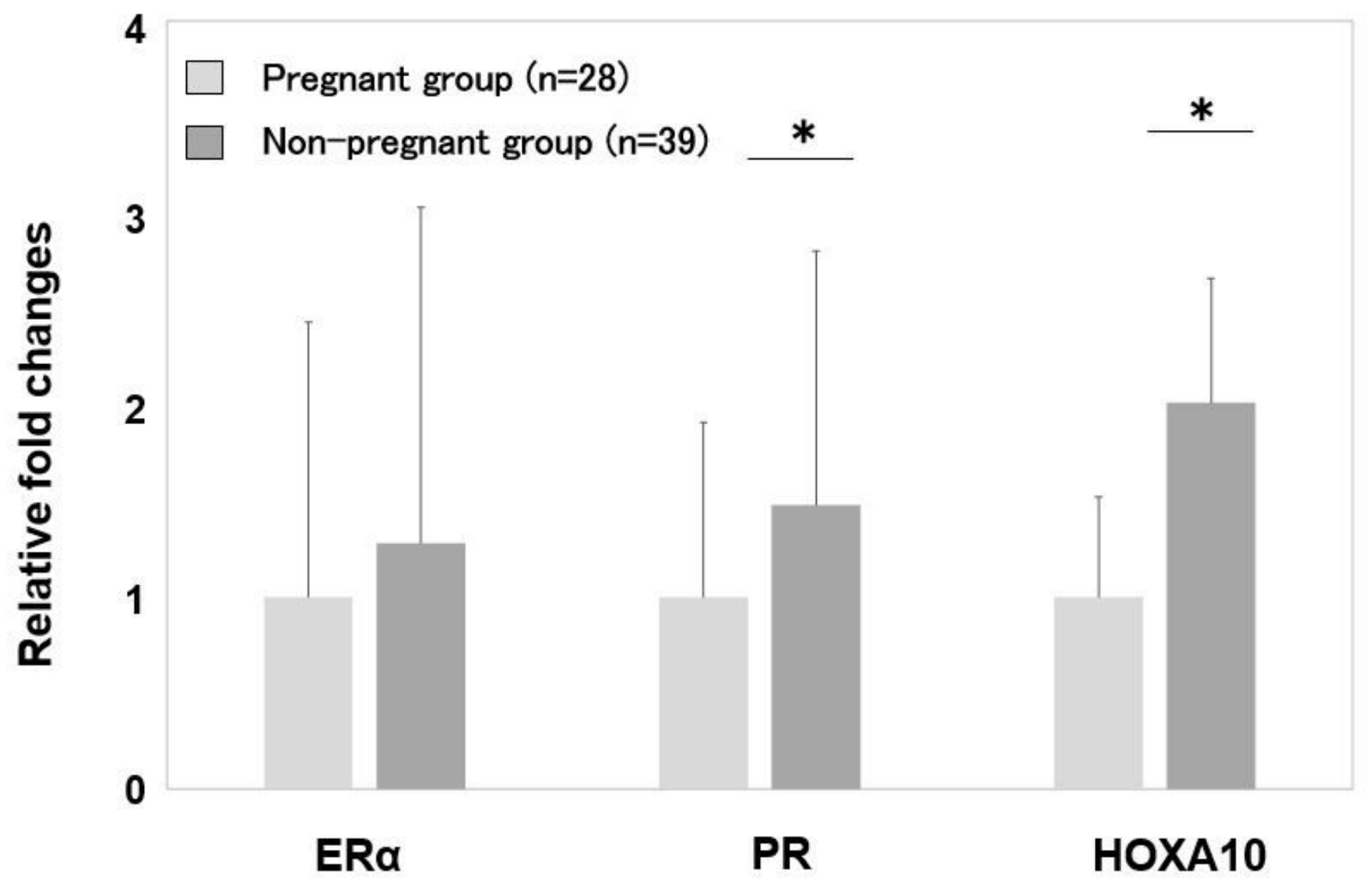

Figure 4

Transcriptional profile in endometrial cells with respect to pregnancy outcomes. The expression of $\mathrm{ERa}$, PR and HOXA10 was determined by RT-qPCR. The fold differences in expression levels were calculated according to the $2-\Delta \Delta \mathrm{Ct}$ method, in duplicates. The data are expressed as mean $\pm \mathrm{SD}$. Bonferroni-Dunn test determined PR and HOXA10 were significantly higher in non-pregnant endometrial cells than those who pregnant $(p<0.05)$.

\section{Supplementary Files}

This is a list of supplementary files associated with this preprint. Click to download.

- Table1.JPG

- Table2.JPG 